\begin{tabular}{|c|l|}
\hline Title & Quasidiffusive propagation of phonons in silicon : Monte Carlo cal culations \\
\hline Author(s) & Tamura, Shin-ichiro \\
\hline Citation & $\begin{array}{l}\text { Physical Review B, 48(18), 13502-13507 } \\
\text { https://doi.org/10.1103/PhysRevB.48.13502 }\end{array}$ \\
\hline Issue Date & 1993-11-01 \\
\hline Doc URL & http://hdl.handle.net/2115/5941 \\
\hline Rights & Copyright $\odot$ 1993 A merican Physical Society \\
\hline Type & article \\
\hline File Information & PRB48-18.pdf \\
\hline
\end{tabular}

Instructions for use 


\title{
Quasidiffusive propagation of phonons in silicon: Monte Carlo calculations
}

\author{
Shin-ichiro Tamura \\ Department of Engineering Science, Hokkaido University, Sapporo 060, Japan \\ (Received 14 December 1992; revised manuscript received 21 June 1993)
}

\begin{abstract}
The time-of-flight spectrum of high-frequency phonons with both anharmonic decay and mass-defect (isotope) scattering is studied numerically for silicon. For the quantitative description of the spectrum in the whole time domain (from $t \simeq t_{b}$ to $t>t_{b}$, where $t_{b}$ is the ballistic time of flight), we make the following substantial improvements beyond previous simulations: (1) For the anharmonic decay we consider, the multibranch decay where the energies and propagation directions of the decayed phonons are determined from the energy-momentum conservation of the process. (2) For the isotope scattering polarization-dependent anisotropic scattering is used when we discuss the phonon intensity at a time $t$ close to $t_{b}$, otherwise an approximate isotropic scattering is employed. (3) The elastic anisotropy is explicitly incorporated through the group velocity of phonons to locate the positions where the scattering events occur. The simulated phonon intensity versus time of flight compares favorably with recent experiments. Specifically, the exponential decay for late arrival times observed experimentally is well reproduced. We also find the phonon focusing due to elastic anisotropy is crucial to explain the shape of the phonon intensity at $t \simeq t_{b}$. The size of the source for the ballistic phonons is also studied.
\end{abstract}

\section{INTRODUCTION}

The time-of-flight spectrum of nonequilibrium phonons injected into nonmetallic crystals at low temperatures generally consists of both ballistic and scattered components. It is well established that the phonon focusing effect $^{1,2}$ due to elastic anisotropy of crystals governs the ballistic phonon propagation. On the other hand, elastic scatterings from mass defects and anharmonic decays (inelastic scatterings) characterize the phonon transport at a time $t$ much later than the ballistic time of flight $t_{b}$. The phonon propagation with both elastic and inelastic scatterings is quasidiffusive. The quantitative understanding of quasidiffusive phonon propagation is very important for analyzing the spectrum of photoexcited phonons ${ }^{3}$ and also for a design of a phonon-based detector of high-energy particles. ${ }^{4,5}$ So far, Levinson and his coworkers have developed an extensive theoretical analysis and have obtained several qualitative results characteristic of the phonon propagation in the presence of both the elastic and inelastic scatterings. ${ }^{6}$ However, it is not so evident whether their results based on a simple model are applicable to direct comparison with the experiments, though both elastic and inelastic processes are experimentally found to be important. ${ }^{7}$

A promising way of understanding quantitatively what happens when high-energy phonons are excited in a crystal would be by the Monte Carlo simulation. Recently, several groups $3,4,8,9$ have made such simulations for studying the time-of-flight spectrum of phonons and also for checking the scaling theory proposed by Kazakovtsev and Levinson. ${ }^{6}$ In their studies, however, a simplified one-branch model where three phonon polarizations are approximated to have the same isotropic spectrum with Debye velocity $v_{D}$ is employed. In addition, anharmonic decay processes are assumed to be collinear. Although we believe that those simulations are useful for understanding qualitatively the characteristic features of quasidiffusive phonon propagation, more quantitative studies, including three distinctive phonon polarizations as well as elastic anisotropy, should be conducted.

In the present study we make more realistic simulations than previous ones by distinguishing between phonon modes. Specifically, for the anharmonic threephonon decays, we explicitly take account of the difference between the longitudinal $(L)$ and transverse $(T)$ phonons and employ the results predicted by the anharmonic elasticity theory. ${ }^{10}$ We also include the anisotropy through the group velocity of phonons to locate the positions where the scattering events occur. This is very important to explain the phonon intensity arriving at the detector at $t \simeq t_{b}$. The lattice dispersion is, however, not taken into account.

\section{ELASTIC AND INELASTIC SCATTERINGS}

We assume a high-purity silicon sample and consider the isotope scattering for an elastic scattering. If the lattice dispersion is neglected, the isotope scattering is isotropic, i.e., the total scattering rate $\Gamma_{I}$ of a phonon is independent of both phonon mode and propagation direction, and given by $\Gamma_{I}=B[E / h(\mathrm{THz})]^{4}$ with $B=2.43 \times 10^{6} \mathrm{~s}^{3}$ for $\mathrm{Si}$, where $E$ is the energy of a phonon and $h$ is the Planck constant. However, an individual scattering process specified by the initial, and scattered-phonon states $\left(\lambda \rightarrow \lambda^{\prime}\right)$ is anisotropic, i.e., it depends on the inner product of the polarization vectors (e) of the initial and final phonons, i.e., $\Gamma_{I}=\sum_{\lambda^{\prime}} \gamma_{\lambda \lambda^{\prime}}$ and $\gamma_{\lambda \lambda^{\prime}} \propto\left|\mathbf{e}_{\lambda} \cdot \mathbf{e}_{\lambda^{\prime}}\right|^{2} / v_{\lambda^{\prime}}^{3}$, where $\lambda=(\mathbf{k}, j)$ denotes the wave vector $\mathbf{k}$ and mode $j$ of a phonon, and $v$ is the phase velocity. Extensive simulations of phonon propagation, including the isotope scattering, have recently been made. ${ }^{11}$ An important observation is that the polarization dependence in an individual scattering is essential for explaining the experimentally observed channeling of a few times scat- 
téred phonons in Si and GaAs. A simple approximation, assuming an isotropic distribution for the scatteredphonon direction, should be a good approximation only for phonons detected at $t$ much later than $t_{b}$.

At low temperatures, the spontaneous splitting of a phonon into two lower energy phonons is the dominant anharmonic decay process of phonons. For these processes, we assume the isotropic approximation where only $L$ phonons can decay via $L \rightarrow L+T$ and $L \rightarrow T+T$ processes. The three-phonon processes in anisotropic solids have recently been calculated for several crystals ${ }^{12-15}$ but the incorporation of the results into the Monte Carlo simulations is very complicated and we do not consider the anisotropy for the decay processes.

The calculation of the decay rate $\Gamma_{A}$ of $L$ phonons in the anharmonic elasticity model has been given in Ref.
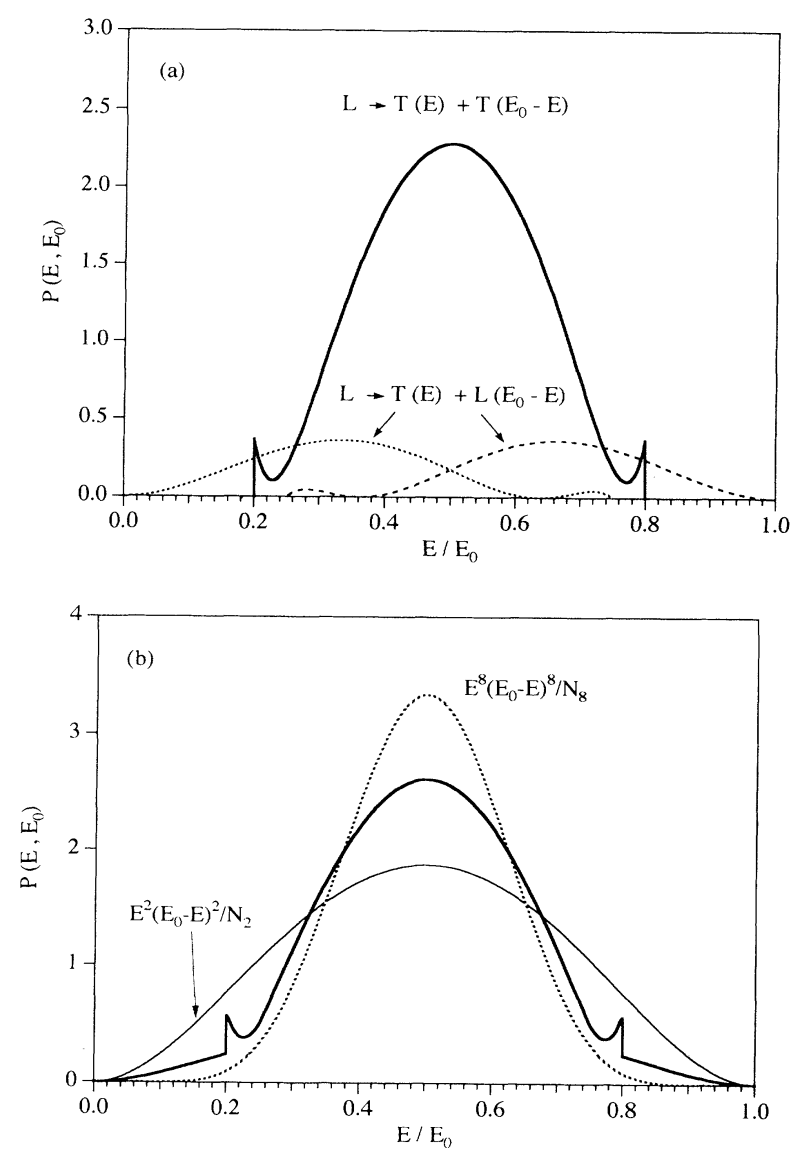

FIG. 1. (a) Energy distribution of daughter phonons produced by the spontaneous decay of an $L$ phonon of energy $E_{0}$ (isotropic approximation). The area surrounded by the solid line (two dashed lines) and the horizontal axis is proportional to the decay rate $\Gamma_{L \rightarrow T+T} \quad\left(\Gamma_{L \rightarrow L+T}\right)$, where $\Gamma_{A}=\Gamma_{L \rightarrow T+T}+\Gamma_{L \rightarrow L+T}$. (b) Comparison of $P\left(E, E_{0}\right)$ used by Maris (Ref. 9) (thin solid line), by Happek et al. (Ref. 22) (dashed line) and in the present model (bold solid line). The areas surrounded by these curves and the horizontal line is normalized to unity, or $\int_{0}^{E_{0}} P\left(E, E_{0}\right) d E=1 . \quad N_{2}=E_{0}^{5} / 30$ and $N_{8}=E_{0}^{17} / 218790$.
10. Explicitly, $\Gamma_{A}=A[E / h(\mathrm{THz})]^{5}$ with $A=7.41$ $\times 10^{4} \mathrm{~s}^{4}$ is obtained for $\mathrm{Si}$ with third-order elastic constants measured at room temperature. ${ }^{16} \mathrm{We}$ plot in Fig. 1 the energy distribution function $P\left(E, E_{0}\right)$ of a daughter phonon in Si produced from the parent of energy $E_{0}$. We see that there are low-energy thresholds for $T$ phonons produced by the decay process $L \rightarrow T+T$ and also for the $L$ phonon produced by the decay process $L \rightarrow L+T$, but no such low-energy threshold exists for the $T$ phonon produced in the latter process. The presence of these thresholds originates from the energy-momentum conservation for the decay processes and has important effects on the time evolution of phonons in the quasidiffusive regime, as we shall see below. Here we note that the relative magnitudes of the areas surrounded by $P\left(E, E_{0}\right)$ and the energy axis correspond to the relative probabilities for the indicated decay processes to occur. For $\mathrm{Si}$, the decay rate for $L \rightarrow T+T$ is about four times larger than that for $L \rightarrow L+T$. $^{10}$

\section{PROCEDURES FOR SIMULATION}

In a simulation, a large number of phonons are generated at a point (the origin) in the bulk of $\mathrm{Si}$ with wave vector $\mathbf{k}$ in a random direction. The initial energy $E_{\text {init }}$ assigned for each phonon is the same and the relative population of the initial phonons of each mode is specified to be proportional to the density of states [0.55:0.35:0.1 for show transverse (ST), fast transverse (FT), and $L$ phonons, respectively]. The primary highenergy acoustic phonons generated by the decay of optical phonons would be $L$ mode but very quick randomization of modes should occur due to strong elastic scatterings. Here, we note that if $E_{\text {init }}$ is sufficiently large ( $E_{\text {init }} / h>4 \mathrm{THz}$, for example), the result is independent of this value as pointed out by Maris. This is because the mean free path limited by the elastic scattering is highly frequency dependent. The phonons can escape from the excitation region and propagate a macroscopic distance (e.g., $1 \mathrm{~mm}$ ) only after being down converted to an energy close to $1.0 \mathrm{THz}$. It should be noted that the elastic mean free path of $1-\mathrm{THz} T$ phonons in $\mathrm{Si}$ is $2.4 \mathrm{~mm}$.

A phonon emitted from the origin begins the process. The total scattering rate $\Gamma_{\text {tot }}=\Gamma_{I}+\Gamma_{A}\left(\Gamma_{\text {tot }}=\Gamma_{I}\right)$ is used to generate the time for the first scattering for $L$ phonons ( $T$ phonons). This scattering for $L$ phonons is either elastic (isotope scattering) or inelastic (anharmonic decay), which is determined according to the relative magnitudes of $\Gamma_{I}$ and $\Gamma_{A}$. For the elastic scattering, the direction of the phonon wave vector after the scattering is given randomly when we study the phonon propagation at $t$ much later than $t_{b}$, but it is given according to the exact formula describing the anisotropic scattering when the behavior of phonons at $t \simeq t_{b}$ is discussed. Note that $T$ phonons are scattered only elastically and do not decay at all.

For the inelastic scattering, the directions and energies of daughter phonons are determined from the energymomentum conservation of the process. (In contrast to the case of the one-branch model, the collinear decays never happen.) The group velocity is used to find the po- 
sition at which a scattering event occurs. After repeating these scattering processes, phonons are assumed to be detected if they first reach the spherical surface of radius $D$ from the origin. So, no surface effect is considered explicitly but this simulation is equivalent to the one where phonons are excited at a point on a flat surface of a hemispherical sample and the phonon reflection at the excitation surface is specular without mode conversion (phonon detection is still on the hemispherical surface). The frequency up conversion which should be important only at the high-excitation levels of phonons is neglected in the present work.

\section{RESULTS OF THE SIMULATIONS}

For comparison, we first present the simulation with the one-branch model proposed by Maris with $v_{D}=5.91 \times 10^{5} \mathrm{~cm} / \mathrm{s}$ for $\mathrm{Si}^{9}{ }^{9}$ Here we note that he used the same $\Gamma_{I}$ for the elastic-scattering rate but the downconversion rate assumed is not the simple $L$ phonon decay rate. The FT phonon decay in anisotropic Si calculated by Berke, Mayer, and Wehner ${ }^{13}$ is also taken into account. More explicitly, the decay rate used is $\Gamma_{A}^{\text {Maris }}=4.1 \times 10^{4}[E / h(\mathrm{THz})]^{5} \mathrm{~s}^{-1}$, which is an average of the $L$ phonon decay rate $\Gamma_{A, L}$ $=1.8 \times 10^{5}[E / h(\mathrm{THz})]^{5} \mathrm{~s}^{-1}$ and the FT phonon decay rate $\Gamma_{\mathrm{A}, \mathrm{FT}}=6.6 \times 10^{4}[E / h(\mathrm{THz})]^{5} \mathrm{~s}^{-1}$ over the whole spectrum of phonons obtained by weighting the density of states. $^{17}$ In addition, Maris assumed $P\left(E, E_{0}\right) \propto E^{2}\left(E_{0}-E\right)^{2}$. This form of $P\left(E, E_{0}\right)$ [see Fig. 1(b)] results from the assumption of collinear decay with a single band (valid for the $L$ phonons in liquid helium) and does not have the low-energy cutoff for daughter phonons.

Figure 2(a) shows the phonon intensity $(I)$ versus arrival time $t$ we have obtained for $D=2.75 \mathrm{~mm}$ with the one-branch model ( $E_{\text {init }} / h=4 \mathrm{THz}$; which is close to the zone-boundary frequency of $\mathrm{T}$ phonons in the [100] direction and also about $\frac{1}{4}$ of the zone-center optical-phonon frequency in $\mathrm{Si}$ ). This time trace looks similar to the original one by Maris for $D=1 \mathrm{~cm}$ and also for the one simulated by Shields et al. for $D=5.5 \mathrm{~mm}$. (Ref. 3) The inset displays the same time trace but in a semilogarithmic scale. Interestingly, the slope for $t>4 t_{b}$ is almost linear in $t$ indicating that the phonon intensity decreases exponentially with respect to $t$ in this time region. This exponential time dependence has not been predicted explicitly by the scaling argument of Kazakovtsev and Levinson, ${ }^{6}$ but recently has been observed in the experimental studies of nonequilibrium phonons in Si produced by low-power excitation at a vacuum interface. ${ }^{3,18}$ (The quasidiffusion of phonons is observed under the vacuum excitation condition but not observed when liquid helium is in contact with the excitation surface of phonons. $)^{3,18}$ If we fit the time trace of Fig. 2(a) for $t>4 t_{b}$ to an exponential function, the magnitude of the slope, or decay constant $a$ [defined by $I \propto \exp \left(-a t / t_{b}\right)$ ] is found to be 0.55 . This value is, however, much larger than $0.30 \mathrm{ob}-$ tained experimentally for the $2.75-\mathrm{mm}$-thick Si sample. ${ }^{18}$ We have checked that the magnitude of $a$ does not change even if we increase the frequency of initial pho- nons $E_{\text {init }} / h$ up to 6 and $8 \mathrm{THz}$.

Figure 2(b) plots the phonon intensity versus $t$ calculated by using an isotropic two-branch model, which is essentially the same as our model but the anisotropy of the lattice is not included at all. (The anharmonic decays are still noncollinear.) The isotropic scattering is assumed for individual isotope-scattering processes. We recognize that the profile of this time trace is quite different from Fig. 2(a) with a one-branch model. Specifically, the ballistic signal is reduced considerably and the maximum of the phonon signal appears at $t$ much later than $t_{b}\left(t>3 t_{b}\right)$. However, the exponential decay can be still seen at $t$ later than $(5-6) t_{b}$ and we find $a=0.30$, which is identical to the experimental value. Although the value of $a$ is consistent with experiment, the profile of the phonon intensity of Fig. 2(b) does not resemble the experiment. The experimental time trace in the [100] direction of Si looks rather like Fig. 2(a) (the simulation with a one-branch model) having a sharp onset of a ballistic signal at $t=t_{b}$, followed by the monotonically decreasing tail. ${ }^{3,18}$ Nevertheless, the shape of the
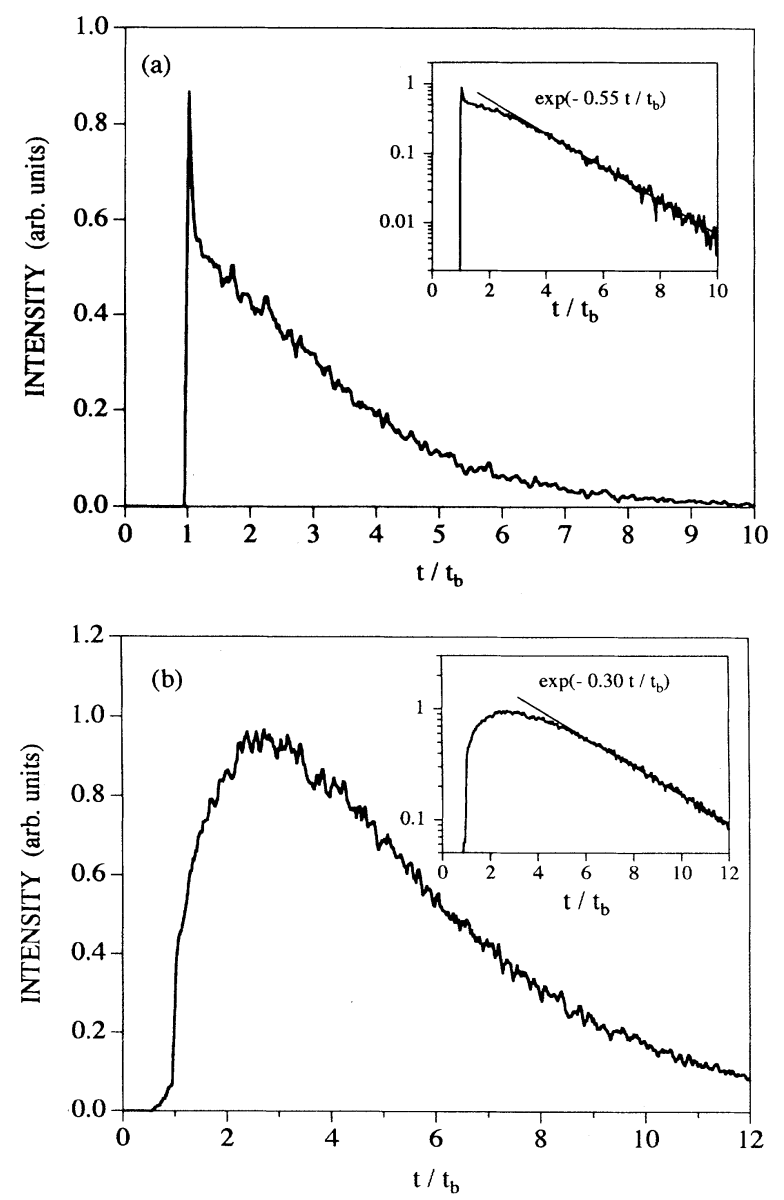

FIG. 2. Time traces of phonon intensity obtained by (a) onebranch model with $P\left(E, E_{0}\right) \propto E^{2}\left(E_{0}-E\right)^{2}$ and (b) isotropic two-branch model. Insets show the same time traces but the intensities are in the semilogarithmic scale. $E_{\text {init }} / h=4 \mathrm{THz}$ and $D=2.75 \mathrm{~mm}$. 
phonon pulses, especially for $t \simeq t_{b}$, should be strongly affected by the phonon focusing effect, so a simulation including the anisotropy is very important.

To this end we have plotted in Fig. 3 the simulated time trace in the [100] direction of $\mathrm{Si}$, based on our model including anisotropy. The overall intensity profile of this figure coincides well with the experimental time trace obtained recently by Msall and Wolfe. ${ }^{18}$ The intensity versus $t$ is fitted to an exponential function over almost the entire region of $t$ plotted $\left(t=t_{b}\right.$ to $\left.10 t_{b}\right)$ and we find $a=0.27$, which is consistent with the experimental value $a=0.30$ for the $2.75-\mathrm{mm}$-thick sample. We also have repeated the same simulation for $5.5-\mathrm{mm}$-thick $\mathrm{Si}$ and found $a=0.24$.

In the simulations we assume that very high-energy phonons $\left(E_{\text {init }} / h=4-8 \mathrm{THz}\right)$ are excited at a point source. The elastic mean free path of the $4 \mathrm{THz} T$ phonon is $9.5 \mu \mathrm{m}$, which is very short compared to $D(1-10$ $\mathrm{mm})$. However, we observe a large signal of ballistic phonons. This result implies that anharmonic decays occur in the vicinity of the point source and low-energy phonons with an elastic mean free path of the order of $D$ emanate from this region. We also have studied the size of the effective source of ballistic phonons, or "the decay spot." A simple argument assuming that the daughter phonons have about half the energy of their parents leads to the diffusion length $\propto E^{-9 / 2}$ for phonons with energy $E$. This suggests that the phonons arriving at the detector originate from the diffusive cloud of their parents, which should be $2^{9 / 2}(=22.6)$ times smaller than the size of $D$.

In Fig. 4 we plot the distribution for the distance $r$ from the origin at which the last scattering event happens for a phonon detected within the time interval $t<1.5 t_{b}$. We see the sharp peak at the origin and its width is close to $2^{-9 / 2} D$. We also plot the pseudo-three-dimensional plot of phonon intensity near the [001] direction detected within the same time interval. Anisotropic spatial distribution due to the phonon focusing is clearly seen. In this

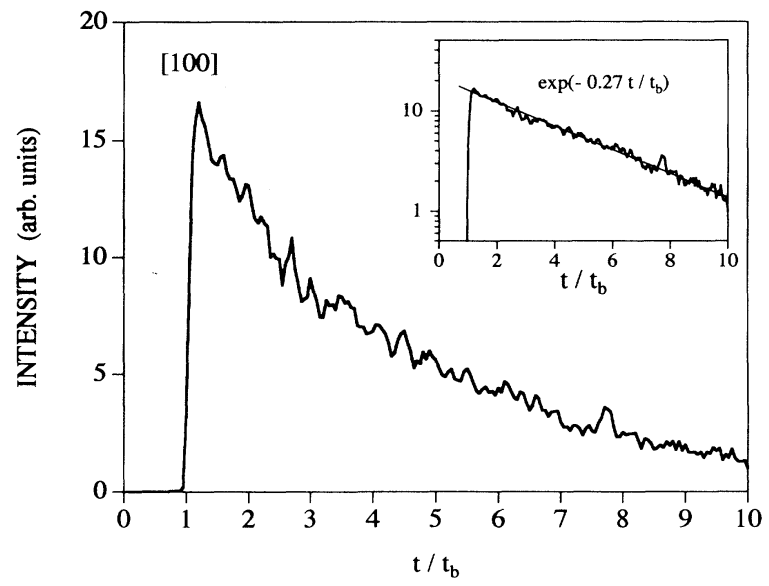

FIG. 3. Time trace of phonon intensity in the [100] direction of $\mathrm{Si}\left(D=2.75 \mathrm{~mm}\right.$ and $\left.E_{\text {init }} / h=4 \mathrm{THz}\right)$ obtained by including anisotropy through the group velocity of phonons. Three branches of phonons are considered but isotropic approximation is used for the anharmonic decay.

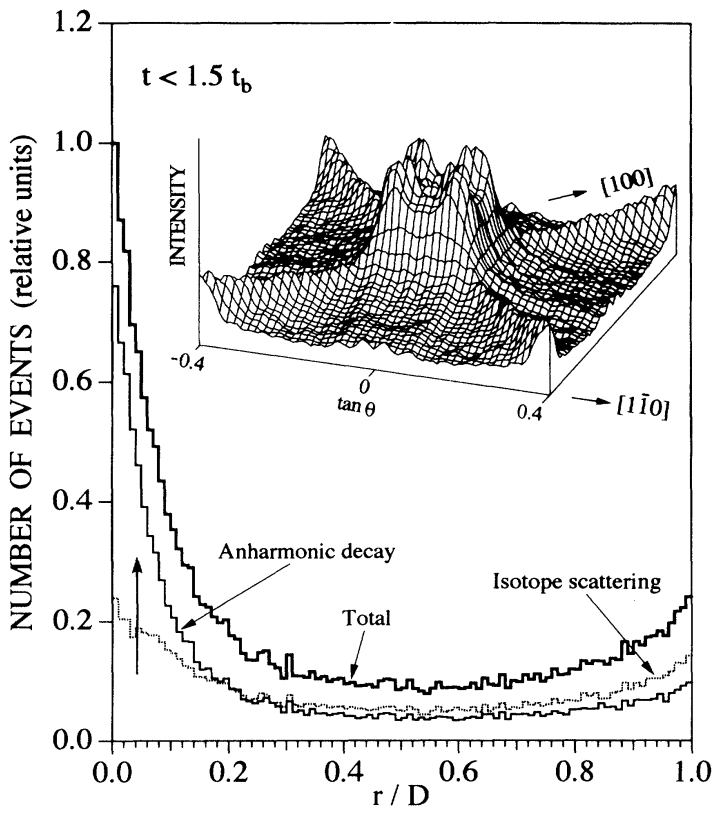

FIG. 4. The distribution of positions at which the last scattering events occur for phonons detected within the time interval $t<1.5 t_{b}$ (bold solid line). The contributions of the anharmonic decay and elastic-scattering events are plotted by a thin solid line and dotted line, respectively. The vertical arrow indicates the position of $r / D=2^{-9 / 2}$. Inset shows the pseudothree-dimensional representation of the phonon intensity detected within the same time interval. (The center is the [001] direction.) $D=1 \mathrm{~cm}$ and $E_{\text {init }} / h=6 \mathrm{THz}$.

simulation $D=1 \mathrm{~cm}$ and $E_{\text {init }} / h=6 \mathrm{THz}$ are chosen, and the anisotropic individual scattering is assumed for the isotope scattering. The energy distribution of detected phonons has a peak at $E=h \times(0.7 \mathrm{THz})$. Here we note that the elastic mean free path of $0.7 \mathrm{THz} T$ phonon is 1 $\mathrm{cm}(=D)$ in silicon.

\section{DISCUSSION}

At present we do not have a physical explanation for the exponential decay of phonon intensity observed in both experiments and simulations for the quasidiffusive propagation regime. In the case of purely diffusive propagation with only elastic scattering, the exponential time dependence of the phonon intensity for $t \gg t_{b}$ is obtained by solving the diffusion equation with an appropriate boundary condition, ${ }^{19,20}$ and we find that agreement of the decay constants deduced from the diffusion equation and the Monte Carlo simulation is excellent. However, the situation is not so simple in the presence of anharmonic decay in addition to elastic scattering, although a possible argument has been developed for the exponential decay by solving the integro-differential equation obeyed by the phonons on the basis of the diffusion approximation. ${ }^{21}$ More extensive studies are needed, both theoretically and experimentally, to resolve this issue.

If the exponential behavior holds for a broader range of conditions, we expect the following results: The mag- 
nitude of the decay constant $a$ should be larger (a) for a shorter sample, (b) for a larger anharmonic decay rate, and (c) for a smaller elastic-scattering rate. Also, the magnitude of $a$ should be larger if a daughter phonon with energy close to the parent phonon (implying that the energy of the other daughter phonon is close to zero) is not produced frequency, i.e., high-energy phonons are removed rapidly from the system. All of these features are consistent with Monte Carlo simulations.

As an illustration we plot in Fig. 5 the time trace obtained with a one-branch model, where the daughter phonons are assumed to have the energies exactly half of their parent phonons, i.e., $P\left(E, E_{0}\right) \propto \delta\left(E-E_{0} / 2\right)$, and other parameters are the same as in Fig. 2(a). This energy distribution is the extreme case of $P\left(E, E_{0}\right)$ which has both the low-energy and high-energy cutoffs in $E$. In comparison to Fig. 2(s), the ballistic signal is diminished and the tail decays more rapidly. The reason is that with this form of $P\left(E, E_{0}\right)$, the probability for the production of very low-energy phonons (which can propagate ballistically through the sample) in a single decay is suppressed and at the same time the production of high-energy phonons which should propagate more diffusively is also suppressed. Consequently, a larger decay constant $(a=0.70)$ is obtained in this simulation.

In the study of transient frequency distributions of anharmonically decaying phonons in $\mathrm{CaF}_{2}$, Happek et al. found that in the context of the one-branch model $P\left(E, E_{0}\right) \propto E^{8}\left(E_{0}-E\right)^{8}$ gives good agreement with their experiment. ${ }^{22}$ This form of $P\left(E, E_{0}\right)$ has effective cutoffs at both low and high energies [see Fig. 1(b)]. So, we have repeated the simulation with the one-branch model and $P\left(E, E_{0}\right)$ given above. The profile of the time trace is very similar to Fig. 5 and the decay constant $a=0.67$ is deduced.

Finally, we should remark that if the one-branch model with the mode-averaged decay probability estimated from only the $L$ phonon decay rate is employed, we obtain the time trace similar to Fig. 1(b) for the two-branch model

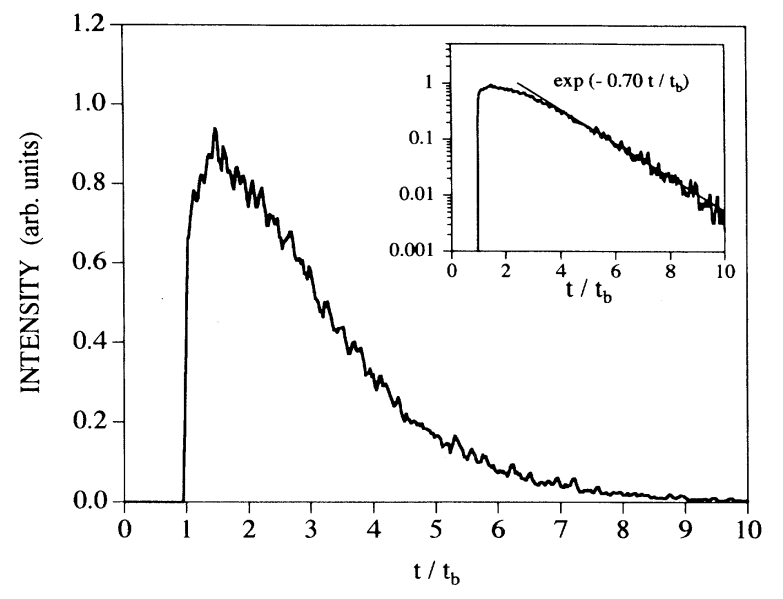

FIG. 5. Time trace obtained by a one-branch model the same as Fig. 2(a) but with $P\left(E, E_{0}\right) \propto \delta\left(E-E_{0} / 2\right)$.

with $\Gamma_{A}$, i.e., the sharp ballistic signal disappears and the tail decays more slowly. Thus, the comparison of the simulations with recent experiments at low-excitation level avoiding the localized phonon source, ${ }^{3,18}$ i.e., "phonon hot spot,"23-26 suggests either the suppression of FT phonon decay or a smaller $L$ phonon decay rate than the theoretical calculations based on the anharmonic elasticity theory. The experimental determination of the inelastic-scattering rates in real crystals remains as an important subject to be done in the near future.

\section{ACKNOWLEDGMENTS}

We are deeply indebted to J. P. Wolfe and M. E. Msall for informing us of their experimental results prior to publication and also for useful comments on the manuscript. We also thank S. E. Esipov for helpful discussions. This work was supported in part by the Iketani Science and Technology Foundations.
${ }^{1}$ B. Taylor, H. Maris, and C. Elbaum, Phys. Rev. Lett. 23, 416 (1969); Phys. Rev. B 3, 1462 (1971).

${ }^{2}$ G. A. Northrop and J. P. Wolfe, in Nonequilibrium Phonon Dynamics, edited by W. E. Bron (Plenum, New York, 1985), p. 165.

${ }^{3}$ J. A. Shields, M. E. Msall, M. S. Carroll, and J. P. Wolfe, Phys. Rev. B 47, 12510 (1993).

${ }^{4}$ B. Cabrera, in Phonons 89, edited by S. Hunklinger, W. Ludwig, and G. Weiss (World Scientific, Singapore, 1990), p. 1373.

${ }^{5}$ B. Sadoulet, B. Cabrera, H. J. Maris, and J. P. Wolfe, in Phonons 89 (Ref. 4), p. 1383.

${ }^{6}$ D. V. Kazakovtsev and Y. B. Levinson, Phys. Status Solidi B 96, 117 (1979); Y. B. Levinson, in Nonequilibrium Phonons in Nonmetallic Crystals, edited by W. Eisenmenger and A. A. Kaplyanski (North-Holland, Amsterdam, 1986), p. 91.

${ }^{7}$ W. E. Bron, Y. B. Levinson, and J. M. O'Connor, Phys. Rev. Lett. 49, 209 (1982); T. E. Wilson, F. M. Lurie, and W. E. Bron, Phys. Rev. B 30, 6103 (1984).
${ }^{8}$ M. Lax, V. Narayanamurti, R. Ulbrich, and N. Holzwarth, in Phonon Scattering in Condensed Matter, edited by W. Eisenmenger, K. Lassmann, and S. Dottinger (Springer, Berlin, 1984 ), p. 103; M. Lax, V. Narayanamurti, R. C. Fulton, and N. Holzwarth, in Phonon Scattering in Condensed Matter V, edited by A. C. Anderson and J. P. Wolfe (Springer, Berlin, 1986), p. 335.

${ }^{9}$ H. J. Maris, Phys. Rev. B 41, 9736 (1990).

${ }^{10}$ S. Tamura, Phys. Rev. B 31, 2574 (1985).

${ }^{11}$ M. T. Ramsbey, J. P. Wolfe, and S. Tamura, Z. Phys. B 73, 167 (1988); J. A. Shields, J. P. Wolfe, and S. Tamura, ibid. 76, 295 (1989); J. A. Shields, S. Tamura, and J. P. Wolfe, Phys. Rev. B 43, 4966 (1991); S. Tamura, J. A. Shields, and J. P. Wolfe, ibid. 44, 3001 (1991); M. T. Ramsbey, S. Tamura, and J. P. Wolfe, ibid. 46, 1358 (1992).

${ }^{12}$ S. Tamura and H. J. Maris, Phys. Rev. B 31, 2595 (1985).

${ }^{13}$ A. Berke, A. P. Mayer, and R. K. Wehner, J. Phys. C 21, 2305 (1988).

${ }^{14}$ M. T. Labrot, A. P. Mayer, and P. K. Wehner, J. Phys. C 1, 
8809 (1989)

${ }^{15}$ H. J. Maris and S. Tamura, Phys. Rev. B 47, 727 (1993).

${ }^{16}$ If this decay rate is averaged over three phonon modes weighted by the phonon density of states, $\Gamma_{A}^{\text {av }}$ $=A^{\text {av }}[E / h(\mathrm{THz})]^{5}$ with $A^{\text {av }}=7.41 \times 10^{3} \mathrm{~s}^{4}$ is obtained. This value is smaller than $\Gamma_{A}^{\text {Maris }}$ used by Maris for the simulation with the one-branch model by a factor of 5.5 (see Sec. IV).

${ }^{17}$ The decay rate $\Gamma_{A, L}$ is different from the $\Gamma_{A}$ we use because for the calculation of $\Gamma_{A, L}$, Berke, Mayer, and Wehner (Ref. 13) used the third-order elastic constants measured at low temperatures. The decay rate is enhanced if we use the third-order elastic constants at low temperatures. This has been noted in Ref. 10 .

${ }^{18}$ M. E. Msall and J. P. Wolfe, in Phonon Scattering in Condensed Matter VII, edited by M. Meissner and R. O. Pohl
(Springer, Berlin, 1993), p. 116.

${ }^{19}$ See, for example, N. N. Lebedev, I. P. Skalskaya, and Y. S. Uflyand, Problems of Mathematical Physics (Prentice-Hall, Englewood Cliffs, NJ, 1965).

${ }^{20}$ M. Lax, V. Narayanamurti, and R. C. Fulton, in Laser Optics of Condensed Matter, edited by J. L. Birmen, H. Z. Cummins, and A. A. Kaplyanskii (Plenum, New York, 1987), p. 229.

${ }^{21}$ S. E. Esipov (unpublished).

${ }^{22}$ U. Happek, Y. Ayant, R. Buisson, and K. F. Renk, Europhys. Lett. 3, 1001 (1987).

${ }^{23}$ C. Hensel and R. C. Dynes, Phys. Rev. Lett. 39, 969 (1977).

${ }^{24}$ M. Greenstein, M. A. Tamor, and J. P. Wolfe, Phys. Rev. B 26, 5604 (1982).

25 J. A. Shields and J. P. Wolfe, Z. Phys. B 75, 11 (1989).

${ }^{26}$ Y. B. Levinson, in Phonons 89 (Ref. 4), p. 1225. 